\title{
Proteinuria Is a Determinant of Quality of Life in Diabetic Nephropathy: Modeling Lagged Effects with Path Analysis
}

\author{
Ken Kelley ${ }^{a}$ O. Tolga Aricak ${ }^{b}$ Robert P. Light ${ }^{c, d}$ Rajiv Agarwal ${ }^{c, d}$ \\ a Inquiry Methodology Program and ${ }^{b}$ Educational Psychology, Indiana University, Bloomington, Ind., \\ 'Division of Nephrology, Department of Medicine, Indiana University School of Medicine, and \\ dRichard L. Roudebush VA Medical Center, Indianapolis, Ind., USA
}

\begin{abstract}
Key Words
Chronic kidney disease - Diabetic nephropathy, quality of life $\cdot$ End-stage renal disease $\cdot$ Glipizide $\cdot$ Kidney disease burden - Mental component score $\cdot$ Physical component score - Pioglitazone - Proteinuria - Type 2 diabetes mellitus • Path analysis
\end{abstract}

\begin{abstract}
Background: Diabetic nephropathy with overt proteinuria often progresses relentlessly to end-stage renal disease (ESRD). Material and Methods: To answer the question whether it is impaired glomerular filtration rate (GFR) or its precursor proteinuria which is more related with multiple domains of health-related quality of life (HRQOL), we measured GFR and proteinuria in 44 patients with type 2 diabetes and overt nephropathy and repeated the measurements after 4 months. 38 patients with ESRD due to diabetic nephropathy served as a control group. We used path analysis to examine the association of baseline proteinuria and GFR with baseline and subsequent HRQOL scales. Results: Compared to patients with ESRD, patients with non-dialysis CKD had Kidney Disease Burden (KDB) that was, on a scale from 0 to $100,19.8$ better $(95 \% \mathrm{Cl} 6.9-32.8)$ ( $p=0.003)$. Mental component score (MCS) did not differ and physical component score (PCS) was worse in non-dialysis CKD patients by 8.5 $(p<0.001)$. Proteinuria at baseline was a predictor of PCS, MCS and KDB score at 4 months, suggesting a lagged effect
\end{abstract}

\section{KARGER \\ Fax +41613061234 \\ E-Mail karger@karger.ch}

www.karger.com
(C) 2007 S. Karger AG, Basel

0250-8095/07/0275-0488\$23.50/0

Accessible online at:

www.karger.com/ajn of proteinuria on HRQOL after controlling for the autoregressive effects. GFR was not shown to have a significant impact on HRQOL. One log unit increase in proteinuria was associated with $3.8(p=0.011)$ fall in PCS, $3.3(p=0.043)$ fall in MCS and $10.6(p=0.006)$ fall in KDB. Conclusion: In patients with advanced diabetic nephropathy, we found that proteinuria has a lagged and profound effect on multiple domains of HRQOL.

Copyright $\odot 2007$ S. Karger AG, Basel

\section{Introduction}

Patients with diabetes mellitus rate their health fair or poor three times as often as healthy people [1]. Among patients with diabetes mellitus, those with multiple comorbidities are particularly susceptible to poor healthrelated quality of life (HRQOL) [2]. Patients with chronic kidney disease (CKD) have lower HRQOL than the general population [3]. Although the time when HRQOL is impaired in relation to the stage of CKD is not known, it is well recognized that patients on dialysis have much lower HRQOL than those in the general population and that dialysis is related to mortality and hospitalization [4].

Proteinuria is a modifiable renal and cardiovascular risk factor that mediates progression of CKD $[5,6]$. The risk of progression of kidney disease, dialysis or death is 
Table 1. Baseline characteristics of the study sample

\begin{tabular}{lccc}
\hline & $\begin{array}{c}\text { CKD not } \\
\text { on dialysis }\end{array}$ & $\begin{array}{c}\text { Hemo- } \\
\text { dialysis }\end{array}$ & $\mathrm{p}$ \\
\hline Age, years & $66 \pm 9$ & $54 \pm 10$ & $<0.001$ \\
Males, \% & $44(100)$ & $41(100)$ & $>0.2$ \\
White, \% & $35(80)$ & $8(20)$ & $<0.001$ \\
Black, \% & $9(20)$ & $33(80)$ & \\
BMI, kg/m ${ }^{2}$ & $32.6 \pm 5.5$ & $28.5 \pm 6.6$ & 0.003 \\
Current smoker, \% & $14(32)$ & $11(27)$ & $>0.20$ \\
Insulin, \% & $28(64)$ & $28(68)$ & $>0.2$ \\
Vascular disease, \% & $28(64)$ & $18(46)$ & 0.11 \\
24-Hour systolic BP, mm Hg & $150 \pm 18$ & $137 \pm 17$ & 0.001 \\
24-Hour diastolic BP, mm Hg & $76 \pm 10$ & $77 \pm 12$ & $>0.2$ \\
24-Hour heart rate, bpm & $69 \pm 11$ & $79 \pm 11$ & $<0.001$ \\
HgbA1c, \% & $7.7 \pm 2.3$ & $\mathrm{NA}$ & $\mathrm{NA}$ \\
BUN, mg/dl & $43.7 \pm 14.0$ & $51.4 \pm 13.8$ & 0.08 \\
Albumin, g/dl & $3.7 \pm 0.4$ & $3.8 \pm 0.5$ & $>0.2$ \\
Hemoglobin, g/dl & $13.1 \pm 2$ & $12.6 \pm 1.3$ & 0.15 \\
GFR, ml/min & $44 \pm 31$ & $\mathrm{NA}$ & $\mathrm{NA}$ \\
Protein/creatinine, g/g & $2.57 \times / \div 2.31$ & $\mathrm{NA}$ & $\mathrm{NA}$ \\
\hline
\end{tabular}

Total sample size is 44 for CKD patients and 41 for hemodialysis patients.

directly related to proteinuria [7]. Whether proteinuria is also related to HRQOL has not been evaluated. Furthermore, if remission of proteinuria is associated with improvement in HRQOL, it offers an opportunity to impact HRQOL with therapies that lower proteinuria. In other words, if remission of proteinuria is associated with improvement in HRQOL, besides modifying cardiovascular risk and progression of renal injury, we can directly make patients feel better. The relationship of glomerular filtration rate (GFR) to HRQOL is controversial. Three large studies have examined the cross-sectional relationship of GFR with HRQOL in patients with CKD. In two of these studies, no relationship was observed between GFR and HRQOL, and in one study there was a relationship. None of these studies used repeated measurements to study longitudinal change.

The purpose of this short-term study in adult men with proteinuria and type 2 diabetes mellitus was to evaluate the impact of directly measured GFR and proteinuria on their impact on HRQOL. We asked the question whether it is GFR or proteinuria that is more related to multiple domains of HRQOL in patients with CKD. To compare the impact of GFR we directly compared baseline level of HRQOL in non-dialysis CKD patients with those with end-stage renal disease (ESRD).

\section{Methods}

\section{Subjects}

We analyzed HRQOL data from 44 patients with type 2 diabetes mellitus who completed a 16-week randomized controlled trial of pioglitazone or glipizide randomly allocated in an equal ratio. The study was blinded to those performing the analyses but open label to the patients and treating physician and has been reported previously [8]. Baseline characteristics of the sample are given in table 1.

Patients with established diabetic nephropathy were recruited from the renal clinic at the Roudebush Veterans Administration Medical Center in Indianapolis, Ind., USA. In order to qualify for inclusion in the study, patients with type 2 diabetes mellitus requiring treatment with oral hypoglycemic drugs or insulin were required to have a urine protein/creatinine ratio of $>1.0 \mathrm{~g} / \mathrm{g}$ on a single voided specimen and a creatinine clearance of $>20 \mathrm{ml} / \mathrm{min}$ by Cockcroft-Gault formula [9]. Exclusion criteria included the presence of liver disease, New York Heart Association Class III or IV heart failure, unstable angina, myocardial infarction or stroke in the previous 3 months, non-steroidal anti-inflammatory drug use, or body mass index of $\geq 40 \mathrm{~kg} / \mathrm{m}^{2}$. The study was approved by the Institutional Review Board of Indiana University and the Research and Development Committee of the Roudebush Veterans Administration Medical Center in Indianapolis and all patients gave their written informed consent.

The primary end-point in the study was reduction in proteinuria, which has been reported elsewhere and is not the subject of this report [8].

\section{Kidney Disease Quality of Life Questionnaire}

The Kidney Disease Quality of Life (KDQOL $\left.{ }^{\mathrm{TM}}\right)$ instrument is a self-report measure that includes a 36-item health survey as the generic core, supplemented with multi-item scales targeted at particular concerns of individuals with kidney disease and on dialysis (symptom/problems, effects of kidney disease on daily life, burden of kidney disease, cognitive function, work status, sexual function, quality of social interaction, sleep). Each scale is scored from 0 to 100 (worst to best) using standard algorithms. The major scales of interest in quality of life were Physical Health Composite Score (PCS), Mental Health Composite Score (MCS) and Kidney Disease Burden (KDB). In the general population, the PCS and MCS have a mean score of 50 each with standard deviation of 10 . The questionnaires were self-administered by study participants at baseline and again at 4 months but were examined for completeness by a research nurse.

\section{Statistical Analysis}

Path analysis is a method used to evaluate a theoretical model with direct and possibly indirect effects between exogenous (predictor/independent) and endogenous (outcome/dependent) variables $[10,11]$. The relationship between variables is quantified with path coefficients, which are much like regression coefficients in a general linear model context. Path coefficients represent the change in an endogenous variable as a function of a 1 unit change in a more exogenous variable that are linked via a path (which represents a direct effect), holding everything else constant. Although path analysis and multiple regressions are similar models, the path analysis model provides a framework for specifying a particular theoretical model about the relationship among a set of 
Fig. 1. Path diagram of proteinuria (a) and GFR (b) on PCS for CKD patients. Singleheaded arrows in the path diagram illustrate the direct effect of one variable on another; the number associated with each of the single-headed arrows is the path coefficient; curved two-headed arrow connecting two variables is the correlation coefficient between the two variables; circles represent the errors in the prediction of the endogenous variables, with the number inside the circle being the standard deviation of the error; $\mathrm{R}^{2}$ represents the proportion of variance in the endogenous variable accounted for by the exogenous variable(s). ${ }^{* *} \mathrm{p}<0.001,{ }^{* *} \mathrm{p}<0.01,{ }^{*} \mathrm{p}<$ 0.05 .

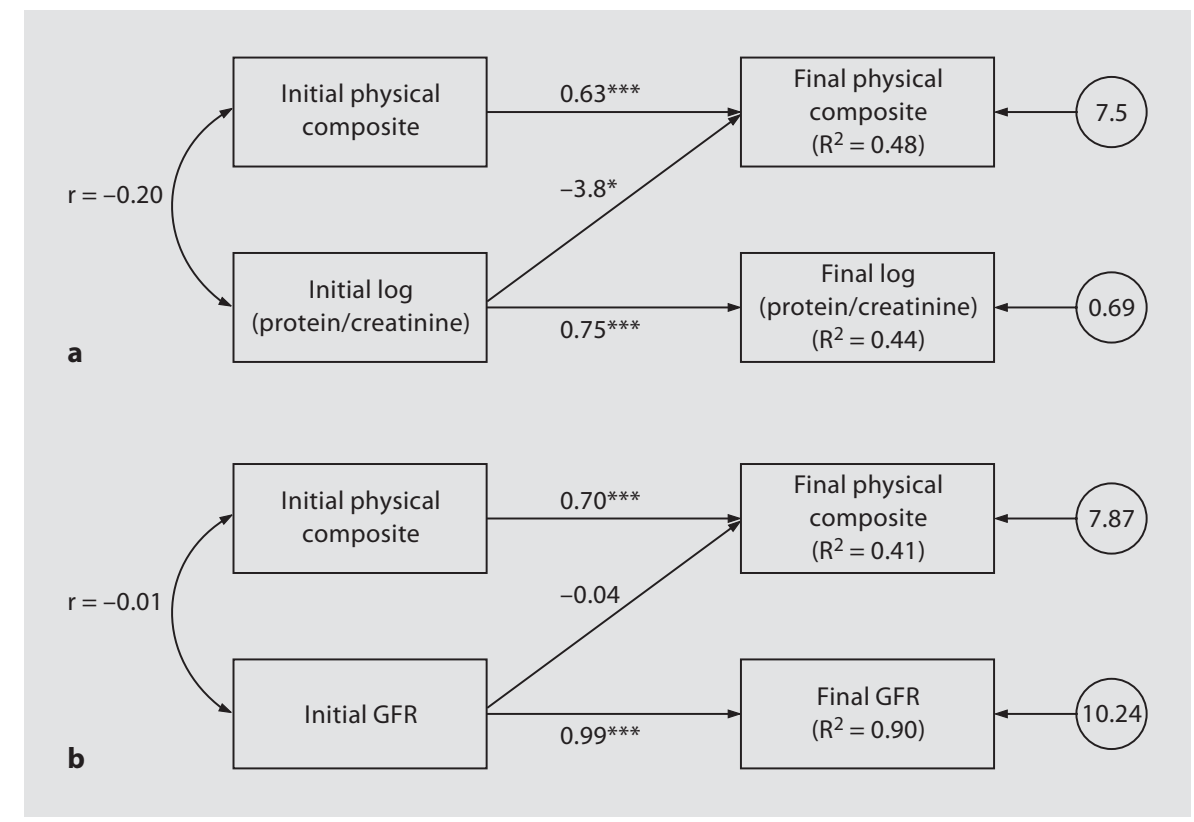

exogenous and endogenous variables. Although path analysis is widely used in some disciplines, to our knowledge, path analysis has been used little in nephrology.

One aspect of evaluating the model is comparing the observed and the model implied covariance matrices to assess general agreement. The goodness of fit of the model can be quantified and referenced to a $\chi^{2}$ distribution, assuming normality and a sufficiently large sample size. A statistically significant $\chi^{2}$ statistic implies that differences exist in the observed and model implied covariance matrices, which provides evidence of model misfit. Another way to evaluate the fit of a model is by using the root mean square error of approximation (RMSEA), with a widely used rule of thumb being values $<0.05$ represent 'good fit' [12]. In addition to evaluating the overall model fit, each of the direct and indirect effects has an estimated path coefficient and a standard error. The path analysis models discussed momentarily were fitted using Amos [13].

GFR was measured with iothalamate clearance and proteinuria by duplicate collections of 24-hour urine protein at baseline and at 4 months. 24-Hour urine protein excretion was calculated per gram urinary creatinine. Results of the two 24-hour urine collections for each visit were averaged to reflect protein excretion rate at baseline and again at the month 4 visits. For the purposes of statistical analysis, the protein excretion rate per gram creatinine was $\log _{\mathrm{e}}$ transformed to better approximate a normal distribution. We applied path analysis models to understand the relationship between MCS, PCS, and KDB as a function of proteinuria and GFR.

Comparisons of (unadjusted) group means were conducted with the ordinary independent groups $t$ tests. Comparisons of initial scores versus final scores for non-dialysis CKD were conducted with the ordinary paired samples $t$ test. Group means were also compared for initial non-dialysis CKD versus hemodialysis, where the mean difference was adjusted for age and ethnicity. The adjusted comparison was performed in an analysis of covariance context, which can be considered an adjusted independent groups $\mathrm{t}$ test since there were only two groups.

We evaluated the relationship of MCS, PCS, and KDB at the final time point using either $\log$ (protein/creatinine) or GFR, while controlling for autoregressive effects (the path between the variable at the initial time and the variable at the final time). These models were fitted separately in a series of six models. Figure 1 reports the specific path diagrams fit with MCS as the outcome variable conditional on (a) $\log$ (protein/creatinine) and (b) GFR. Figures 2 and 3 are analogous to figure 1 with PCS and KDB replacing MCS, respectively. Less the path analysis, the MBESS [14] $\mathrm{R}$ package [15] and SPSS [16] were used, as appropriate, for the specific analyses that follow.

\section{Results}

\section{Study Participants}

Fifty-four subjects qualified and consented to participate in the study, and 44 were randomized to treatment. This report is limited to the 44 patients who were randomized and had repeated measurements of GFR, proteinuria and HRQOL. Actual subject study participation occurred between November 2001 and December 2003. Two subjects withdrew following randomization, 1 under advice of a primary care physician, and the other following hospitalization for a gastrointestinal bleed. One subject died due to metastatic adenocarcinoma. Finally, 1 subject was unable to present for the final study visit. Thus, 40 subjects - 19 in the glipizide arm and 21 in the pioglitazone arm - completed the study. 
Fig. 2. Path diagram of proteinuria (a) and GFR (b) on MCS for CKD patients. ${ }^{* * *} \mathrm{p}<$ $0.001,{ }^{* *} \mathrm{p}<0.01,{ }^{*} \mathrm{p}<0.05$.

Fig. 3. Path diagram of proteinuria (a) and GFR (b) on KDB for CKD patients. ${ }^{* * *} \mathrm{p}<$ $0.001,{ }^{* *} \mathrm{p}<0.01,{ }^{*} \mathrm{p}<0.05$.
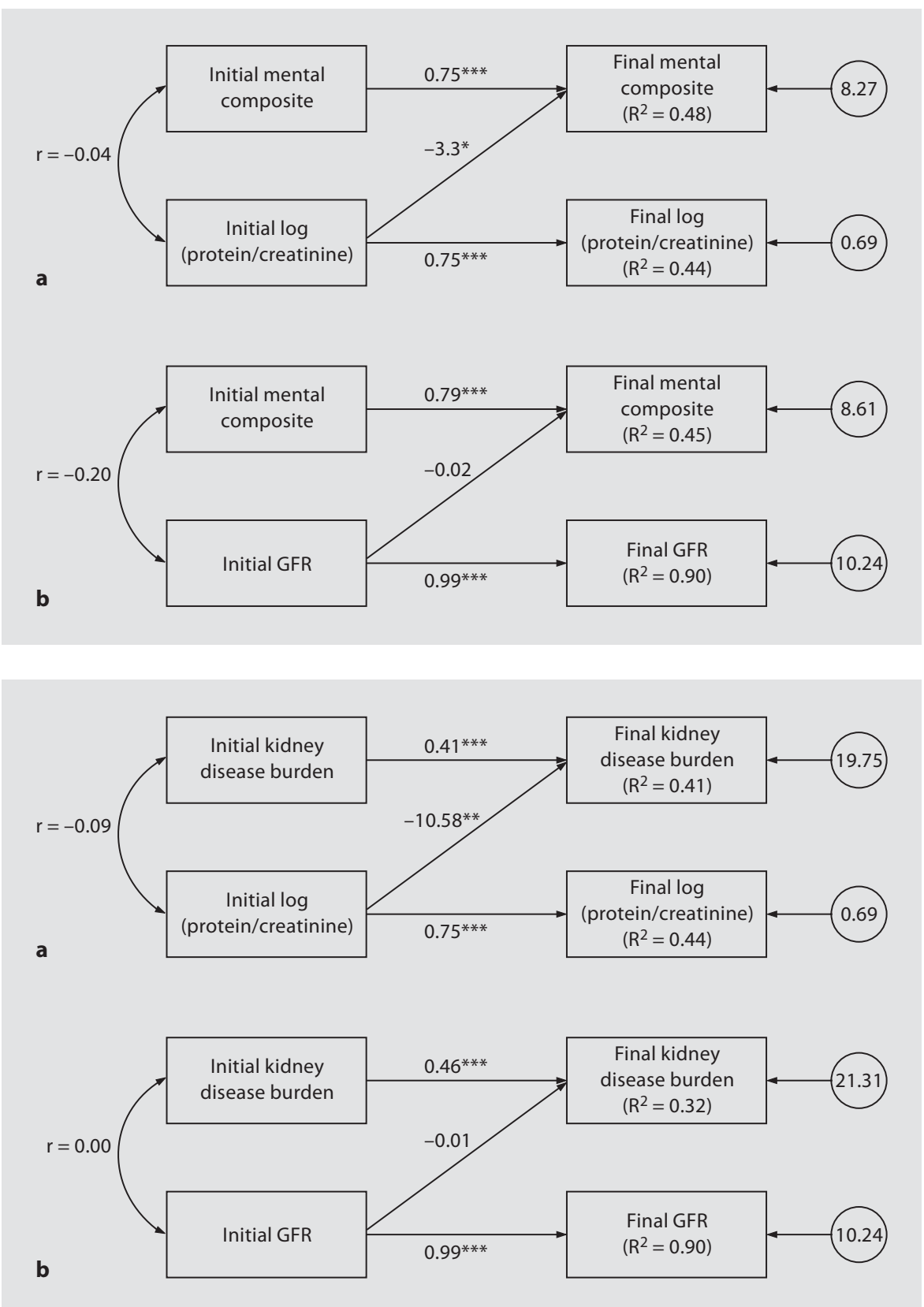

Baseline characteristics of patients with diabetic nephropathy not on dialysis and those with ESRD secondary to diabetes mellitus on hemodialysis are shown in table 1. All but 8 patients were taking an ACE inhibitor or an angiotensin receptor blocker. ESRD patients were younger and more African-American.

The relationship of HRQOL domains with CKD and ESRD is shown in table 2. It is clear that patients with ESRD due to diabetes have worse KDB by nearly 20 points compared to those not on dialysis, which implies a standardized mean difference of 0.69 (95\% CI $0.24-$ 1.14). However, those on dialysis had a mean PCS of 8.5 points better than CKD (95\% CI 3.5-13.2). The MCS was similar between the two groups ( 46.8 for CKD and 46.6 for ESRD) and did not differ significantly after adjusting for ethnicity and age. After adjusting for ethnicity and age, KDB was 15.9 worse in ESRD (95\% CI -33.1 to 1.4; $\mathrm{p}=0.071)$ and PCS 7.3 better in ESRD (95\% CI 1.2-13.4). Neither age nor ethnicity was significant for any of the models. 
Table 2. Impact of CKD stage on KDQOL subscales

\begin{tabular}{|c|c|c|c|c|c|}
\hline & \multicolumn{2}{|c|}{ CKD, non-ESRD } & \multirow[t]{2}{*}{ Hemodialysis } & \multirow{2}{*}{$\begin{array}{l}\text { Difference }(95 \% \mathrm{CI}) \\
\text { CKD initial vs. HD }\end{array}$} & \multirow{2}{*}{$\begin{array}{l}\text { Adjusted difference }(95 \% \mathrm{CI})^{1} \\
\text { CKD initial vs. HD }\end{array}$} \\
\hline & initial & final & & & \\
\hline Mental Composite Score & $46.8 \pm 10.0$ & $48.2 \pm 11.8$ & $46.6 \pm 7.8$ & $-0.13(-4.27$ to 4.02$)$ & $2.7(-2.7$ to 8.1$)$ \\
\hline Physical Composite Score & $33.7 \pm 9.5$ & $33.8 \pm 10.5$ & $42.2 \pm 10.9$ & $8.5(3.5 \text { to } 13.2)^{* * *}$ & $7.3(1.2 \text { to } 13.4)^{*}$ \\
\hline Kidney Disease Burden & $67.6 \pm 32.3$ & $64.9 \pm 26.0$ & $47.8 \pm 23.8$ & $-19.8(-6.9 \text { to }-32.8)^{* *}$ & $-15.9(-33.1$ to 1.4$)(\mathrm{p}=0.071)$ \\
\hline
\end{tabular}

Scores are on a 100-point scale where 0 is worst and 100 is best.

${ }^{*} \mathrm{p}<0.05 ;{ }^{* *} \mathrm{p}<0.01 ;{ }^{* * *} \mathrm{p}<0.001$; hemodialysis vs. CKD initial.

${ }^{1}$ Adjusted for age and ethnicity (neither of which was significant for the three analyses).

Table 3. Pearson zero-order correlation coefficients for the CKD patients between KDQOL subscales and GFR/proteinuria

\begin{tabular}{|c|c|c|c|c|}
\hline & \multicolumn{2}{|c|}{$\log ($ protein/creatinine $)$} & \multicolumn{2}{|l|}{ GFR } \\
\hline & initial & final & initial & final \\
\hline \multicolumn{5}{|c|}{ Mental Composite Score } \\
\hline Initial & -0.03 & 0.023 & -0.21 & -0.08 \\
\hline Final & $-0.29, p=0.607$ & -0.07 & -0.07 & 0.06 \\
\hline \multicolumn{5}{|c|}{ Physical Composite Score } \\
\hline Initial & -0.20 & $-0.30, \mathrm{p}=0.071$ & -0.01 & -0.02 \\
\hline Final & $-0.41^{* *}$ & $-0.33^{*}$ & -0.14 & -0.09 \\
\hline \multicolumn{5}{|c|}{ Kidney Disease Burden } \\
\hline Initial & -0.09 & -0.10 & 0.00 & 0.02 \\
\hline Final & $-0.40^{* *}$ & $-0.27, \mathrm{p}=0.095$ & -0.01 & 0.08 \\
\hline \multicolumn{5}{|c|}{$\log ($ protein/creatinine $)$} \\
\hline Initial & 1.00 & $0.64^{* * *}$ & -0.24 & $-0.32, \mathrm{p}=0.051$ \\
\hline Final & $0.64^{* * *}$ & 1.00 & $-0.42^{* *}$ & $-0.40^{*}$ \\
\hline \multicolumn{5}{|l|}{ GFR } \\
\hline Initial & -0.24 & $-0.42^{* *}$ & 1.00 & $0.95^{* * *}$ \\
\hline Final & $-0.32, p=0.051$ & $-0.40^{*}$ & $0.95^{* * *}$ & 1.00 \\
\hline
\end{tabular}

MCS, PCS and KDB are based on a 0 - to 100 -point scale, where 0 is worst and 100 is best. Probability values are based on the $t$ test for Pearson zero-order correlation coefficients.

${ }^{*} \mathrm{p}<0.05 ;{ }^{* *} \mathrm{p}<0.01 ;{ }^{* *} \mathrm{p}<0.001$ (all two-tailed).
The zero-order Pearson product moment correlation coefficients between PCS, MCS and KDB versus GFR and proteinuria for the CKD patients are shown in table 3. Weak and non-significant correlations are seen between GFR and HRQOL subscales. On the other hand, significant relationships emerged between final HRQOL subscale scores and baseline $\log _{\text {e }}$ protein/creatinine ratio (which is consistent with the path for the lagged effect of proteinuria at baseline and HRQOL at the final time).

The six path analysis models are represented in figures $1-3$. The single-headed arrows in the path diagrams illustrate the direct effect of an exogenous variable (proteinuria or GRF) on an endogenous variable (MCS, PCS, or KDB). The number associated with each of the singleheaded arrows is the path coefficient that quantifies the change in the endogenous variable as a function of a 1 unit change in the exogenous variable. The curved twoheaded arrow connecting two variables (i.e., the baseline exogenous variables) is the correlation coefficient between the two variables. The circles represent the errors in the prediction of the endogenous variables, with the number inside the circle being the standard deviation of the error. Notice that an $\mathrm{R}^{2}$ value is given for each of the endogenous variables. The value of $\mathrm{R}^{2}$ represents the proportion of variance in the endogenous variable accounted for by the exogenous variable(s) given the model speci- 
fied, which is analogous to the squared multiple correlation coefficient in a general linear model context. In fact, if final GFR or final proteinuria were removed from the path models, the path models would literally become multiple regression models, which is a special case of a path model.

Baseline GFR did not significantly correlate with baseline HRQOL domains or predict final HRQOL domains. However, baseline proteinuria was a predictor of final PCS, MCS and KDB. There was no relationship of baseline proteinuria and baseline PCS, MCS and KDB, suggesting a lagged effect of final HRQOL conditional on baseline proteinuria. In other words, the effect of a change in proteinuria may not be immediate, but rather requires the passage of time for the effect to manifest. A $1 \log$ unit increase in proteinuria was associated with $-3.8(\mathrm{p}=$ $0.011)$ change in PCS, $-3.3(\mathrm{p}=0.043)$ in MCS and -10.6 $(\mathrm{p}=0.006)$ change in KDB.

Each of the models fit well, with the estimated RMSEA being $<0.05$ for each of the path models and $\chi^{2}$ goodness of fit was not significant for any of the models.

\section{Discussion}

The major findings of our study are that proteinuria profoundly impacts all major domains of HRQOL in a dose-dependent manner with a lagged effect. GFR on the other hand had little impact on HRQOL.

The impact of GFR in cross-sectional studies of HRQOL has been a subject of debate. For example, in the African-American Study of Kidney Disease, KDQOL was administered to 1,094 African-American men and women. Mean GFR was $46 \mathrm{ml} / \mathrm{min}$, PCS was $43.4 \pm 10.9$ and MCS was $51.3 \pm 10.3$. Both scores were higher in men than in women, but neither was related to GFR [17]. On the other hand, in the Modification of Diet in Renal Disease, in 1,284 patients the magnitude of HRQOL and symptoms were negatively correlated with GFR [18]. Finally, in the cross-sectional analysis of the Renal Research Institute Study, GFR was not significantly association with HRQOL [19]. None of the studies reported the association of HRQOL with proteinuria. Since patients participating in the MDRD study were more proteinuric than the AASK study, it is possible that the confounding effect of proteinuria on HRQOL was missed. In a longitudinal study of patients with type 1 diabetes mellitus, microalbuminuria was not associated with impaired HRQOL [20]. Thus, it appears that overt albuminuria, especially in the nephrotic range, may be related to im-

Proteinuria as QOL Determinant paired HRQOL. Although a longer period of many observations would be helpful for evaluating this hypothesis, a randomized controlled trial would be necessary to definitively show support for this hypothesis. Thus, a causeand-effect relationship cannot be established.

In the general population, PCS and MCS averages 50 and has a standard deviation of 10 [21]. The average PCS in non-ESRD patients was 33.7 and MCS was 46.8. PCS in the Renal Research Institute-CKD study was 37.3 slightly higher than our sample, but nearly 1.3 standard deviations below the general population, which is considered substantial impairment [19]. Thus, a much greater impairment in PCS is imposed by CKD at a stage that predates dialysis. CKD induced by diabetes mellitus may have a greater adverse effect on HRQOL compared to CKD induced by other causes [22]. For example, compared to patients with hypertension-induced CKD participating in the AASK study who had a similar GFR, the PCS in our sample was lower by 9.7 units (95\% CI 6.3$13.1), \mathrm{p}<0.001$, and MCS was lower by 4.5 units $(95 \% \mathrm{CI}$ $1.3-7.8), p=0.006$ [17]. In patients starting dialysis, those with type 2 diabetes mellitus have a lower HRQOL compared to those without diabetes [23]. Ethnicity may have an independent effect on HRQOL African-Americans appear to enjoy a greater HRQOL. Table 1 shows that ESRD patients were younger and more African-American. Whether impairment in PCS in our study was due to an independent effect of diabetes mellitus or it was due to fewer African-Americans or older age is not clear [24].

Compared to patients not on dialysis, patients with ESRD had KDB that was nearly 20 points higher. This is not surprising given the burden imposed on the patient by renal replacement therapy. Somewhat unexpectedly, patients on dialysis had a higher PCS compared to those not on dialysis. This may be due to survival bias. PCS is strongly related to mortality, an effect that may manifest before the onset of ESRD $[4,25]$. Thus, those with poor PCS may have died leaving those with higher PCS on dialysis. An alternative though less likely explanation for this finding is that dialysis improved PCS in those with CKD.

The strengths of our study are the direct measurements of GFR, and duplicate measurements of 24-hour urine protein collection at each clinic visit and excellent adherence to protocol. Limitations of our study are the relatively short follow-up and limited number of subjects. Patients with CKD and ESRD had different proportional ethnicities: $80 \%$ of the CKD patients were white, while $80 \%$ of the ESRD patients were black. Adjustment for 
these different ethnicities did not alter the results. The results of our study apply to only men, since we did not study women. It is conceivable that a longer duration or recruiting a larger number of subjects or both would make the association of proteinuria with HRQOL even more clinically relevant, or possibly to show an association between GFR and HRQOL.
In summary, in this single-center study in patients with advanced type 2 diabetic nephropathy we found a greater impact of proteinuria on multiple domains of HRQOL compared to directly measured GFR. Future studies should address the impact of reduction in proteinuria on HRQOL. If a causal relationship is confirmed, proteinuria reduction may not only impact the time to dialysis and cardiovascular events but also quality of life in patients with diabetic nephropathy.

\section{References}

1 CDC: Self-rated fair or poor health among adults with diabetes - United States, 19962005. MMWR Morb Mortal Wkly Rep 2006; 55:1224-1227.

2 Maddigan SL, Feeny DH, Majumdar SR, Farris $\mathrm{KB}$, Johnson JA: Understanding the determinants of health for people with type 2 diabetes. Am J Public Health 2006;96:1649_ 1655.

3 Unruh ML, Weisbord SD, Kimmel PL: Health-related quality of life in nephrology research and clinical practice. Semin Dial 2005; 18:82-90.

4 Mapes DL, Lopes AA, Satayathum S, McCullough KP, Goodkin DA, Locatelli F, Fukuhara S, Young EW, Kurokawa K, Saito A, Bommer J, Wolfe RA, Held PJ, Port FK: Health-related quality of life as a predictor of mortality and hospitalization: the Dialysis Outcomes and Practice Patterns Study (DOPPS). Kidney Int 2003;64:339-349.

5 Hunsicker LG, Adler S, Caggiula A, England BK, Greene T, Kusek JW, Rogers NL, Teschan PE: Predictors of the progression of renal disease in the Modification of Diet in $\mathrm{Re}$ nal Disease Study. Kidney Int 1997;51: 1908-1919.

6 Keane WF, Brenner BM, de Zeeuw D, Grunfeld JP, McGill J, Mitch WE, Ribeiro AB, Shahinfar S, Simpson RL, Snapinn SM, Toto R: The risk of developing end-stage renal disease in patients with type 2 diabetes and nephropathy: the RENAAL study. Kidney Int 2003;63:1499-1507.

7 De Zeeuw D, Remuzzi G, Parving $\mathrm{HH}$, Keane WF, Zhang Z, Shahinfar S, Snapinn S, Cooper ME, Mitch WE, Brenner BM: Proteinuria, a target for renoprotection in patients with type 2 diabetic nephropathy: lessons from RENAAL. Kidney Int 2004;65: 2309-2320.
8 Agarwal R, Saha C, Battiwala M, Vasavada N, Curley T, Chase SD, Sachs N, Semret MH: A pilot randomized controlled trial of renal protection with pioglitazone in diabetic nephropathy. Kidney Int 2005;68:285-292.

9 Cockcroft DW, Gault MH: Prediction of creatinine clearance from serum creatinine. Nephron 1976;16:31-41.

10 Loehlin JC: Latent Variable Models: An Introduction to Factor, Path, and Structural Equation Analysis, ed 4. Mahwah/NJ, Erlbaum Associates, 2004.

11 Kline RB: Principles and Practice of Structural Equation Modeling, ed 2. New York, Guilford Press, 2005.

12 Browne MW, Cudeck R: Alternative ways of assessing model fit. Sociol Methods Res 1992;21:230-258.

13 Arbucke J: Amos User's Guide (Computer Software and Manual), Version 6.0. Amos Development Corp, 2005.

14 Kelley K: Methods for the Behavioral, Educational, and Social Sciences: an R Package (Computer Software and Manual), Version 0.0.8. Retrievable from http://cran.r-project. org, 2007.

15 Development Core Team R: A language and environment for statistical computing. $\mathrm{R}$ Foundation for Statistical Computing, Vienna, Austria. ISBN 3-900051-07-0. Retrievable from http://cran.r-project.org, 2007.

16 SPSS (Computer Software and Manual), Version 15. Chicago, SPSS Inc, 2005.

17 Kusek JW, Greene P, Wang SR, Beck G, West D, Jamerson K, Agodoa LY, Faulkner M, Level B: Cross-sectional study of health-related quality of life in African-Americans with chronic renal insufficiency: the AfricanAmerican Study of Kidney Disease and Hypertension Trial. Am J Kidney Dis 2002;39: 513-524.
18 Rocco MV, Gassman JJ, Wang SR, Kaplan RM: Cross-sectional study of quality of life and symptoms in chronic renal disease patients: the Modification of Diet in Renal Disease Study. Am J Kidney Dis 1997;29:888896.

19 Perlman RL, Finkelstein FO, Liu L, Roys E, Kiser M, Eisele G, Burrows-Hudson S, Messana JM, Levin N, Rajagopalan S, Port FK, Wolfe RA, Saran R: Quality of life in chronic kidney disease: a cross-sectional analysis in the Renal Research Institute-CKD Study. Am J Kidney Dis 2005;45:658-666.

20 Huang GH, Palta M, Allen C, LeCaire T, D'Alessio D: Self-rated health among young people with type 1 diabetes in relation to risk factors in a longitudinal study. Am J Epidemiol 2004; 159:364-372.

21 Ware JE Jr: SF-36 health survey update. Spine 2000;25:3130-3139.

22 Valderrabano F, Jofre R, Lopez-Gomez JM: Quality of life in end-stage renal disease patients. Am J Kidney Dis 2001;38:443-464.

23 Martinez-Castelao A, Gorriz JL, Garcia-Lopez F, Lopez-Revuelta K, De AF, Cruzado JM: Perceived health-related quality of life and comorbidity in diabetic patients starting dialysis (CALVIDIA study). J Nephrol 2004; 17:544-551

24 Unruh M, Miskulin D, Yan G, Hays RD, Benz R, Kusek JW, Meyer KB: Racial differences in health-related quality of life among hemodialysis patients. Kidney Int 2004;65: 1482-1491.

25 Knight EL, Ofsthun N, Teng M, Lazarus JM, Curhan GC: The association between mental health, physical function, and hemodialysis mortality. Kidney Int 2003;63:1843-1851. 